\title{
miR-423-5p Inhibits Osteosarcoma Proliferation and Invasion Through Directly Targeting STMN1
}

\author{
Xuesong Wang ${ }^{a} \quad$ Lei Peng $^{b} \quad$ Xiaojin Gong ${ }^{a} \quad$ Xiugong Zhang ${ }^{a} \quad$ Ruifu Sun ${ }^{a}$ \\ Jinlong $\mathrm{Du}^{\mathrm{a}}$ \\ aSpinal Department of Qingdao Central Hospital, Qingdao Central Hospital, Qingdao, bLibrary of \\ Qingdao Central Hospital, Qingdao Central Hospital, Qingdao, China
}

\section{Key Words}

Osteosarcoma $\cdot$ MicroRNAs $\cdot \operatorname{miR}-423-5 p \cdot S T M N 1$

\begin{abstract}
Background/Aims: Increasing evidences suggest that dysregulated expression of miRNAs contributes to the progression of various tumors. However, the underlying function of miR423-5p in osteosarcoma remains unexplored. Methods: The expression of miR-423-5p and STMN1 were determined in osteosarcoma samples and cell lines via quantitative real-time PCR. Colony formation and Cell Counting Kit-8 (CCK-8) assays were performed to measure cell proliferation ability and transwell analysis was used to detect cell invasion, and dual luciferase reporter assay was perform to analysis the interaction between the miR-423-5p and STMN1. Results: The expression levels of miR-423-5p and STMN1 in the osteosarcoma tissues and cell lines were measured by qRT-PCR. Cell viability was determined using the clone formation and CCK-8 assays. A dual-luciferase reporter and Western blot were performed to stdudy the target gene of miR-423-5p. Here, we showed that miR-423-5p expression was downregulated in osteosarcoma tissues and cell lines. However, the expression of stathmin1 (STMN1) was downregulated in osteosarcoma tissues and cell lines. Moreover, STMN1 expression level was negatively correlated with the miR-423-5p expression in the osteosarcoma tissues. We identified STMN1 was a direct target gene of miR-423-5p in osteosarcoma cell. Overexpression of miR423-5p inhibited osteosarcoma cell proliferation, colony formation and invasion. Furthermore, we demonstrated that STMN1 was involved in miR-423-5p-mediated cell behavior such as cell proliferation, colony formation and invasion in the osteosarcoma cell. Conclusion: Our present study indicated that miR-423-5p acted as a tumor suppressor gene in osteosarcoma partly through inhibiting STMN1 expression.
\end{abstract}




\section{Cellular Physiology Cell Physiol Biochem 2018;50:2249-2259 \begin{tabular}{ll|l} 
DOl: 10.1159/000495085 & (0) 2018 The Author(s). Published by S. Karger AG, Basel \\
and Biochemistry. & Published online: 13 November 2018 & www.karger.com/cpb
\end{tabular} \\ Wang et al.: miR-423-5p Suppressed Osteosarcoma Proliferation and Invasion}

\section{Introduction}

Osteosarcoma is the most prevalent malignant tumor of bone and is usually observed in young adults and adolescents [1-5]. Despite the recent advances in the treatment of osteosarcoma such as radiation therapy, adjuvant chemotherapy and surgery that have been achieved, the relative 5-year survival rate of osteosarcoma patients remains discontent [6-10]. Although various studies have investigated the development and mechanism of osteosarcoma, the molecular mechanism of osteosarcoma is still elusive [11-13]. Therefore, it is urgent to find novel therapeutic approaches and targets for treating osteosarcoma.

MicroRNAs (miRNAs) are an abundant class of small, endogenous, single-stranded and noncoding RNAs that modulate gene expression posttranscriptionally through binding to the 3'UTR (3'-untranslated region) of the target gene [14-17]. MiRNAs participate in many biological processes including cell apotosis, development, proliferation, differentiation, fat metabolism, migration and stress resistance [18-21]. Increasing evidences demonstrated that deregulation of miRNAs could be implicated in a lot of tumors such as hepatocellular carcinoma, glioblastoma, colorectal carcinoma, ovarian carcinoma, renal cell carcinoma and gastric cancer [22-27]. Especially, some miRNAs have been demonstrated to play crital roles in pathogenesis of osteosarcoma [28-30].

The present study aimed to investigate the role of miR-423-5p in osteosarcoma. We showed that miR-423-5p expression was decreased in osteosarcoma tissues and cell lines. We identified STMN1 as a direct target gene of miR-423-5p in the osteosarcoma cell. Overexpression of miR-423-5p suppressed the osteosarcoma cell proliferation, colony formation and invasion.

\section{Materials and Methods}

\section{Tumor Specimens, cell lines and cell tranfection}

Thirty paired osteosarcoma tissues and no-tumor specimens were collected from osteosarcoma patients at our hospital. These tissues were immediately kept in the liquid nitrogen until use. This study was approved with the Ethics Committee of Qingdao Central Hospital and human tissues for this research were collected with informed consent. The osteosarcoma cells (Saos-2, HOS, MG-63 and U2OS) and one normal osteoblast cell line were purchased from the Cell Source of the Academy of Sciences (Shanghai, China). These cells were cultured in the DMEM (Dulbecco's modified Eagle's medium, Invitrogen, USA) supplemented with FBS, penicillin and streptomycin. MiR-423-5p mimic and scramble were purchased from Ribobio (Guangzhou, China). Cell transfection was performed by using the Lipofectamine 2000 (Invitrogen, USA) in accordance to the instruction's information.

\section{qRT-PCR}

Total RNAs from cells or tissues were isolated with Trizol reagent in accordance with manufacturer's instruction. For miR-423-5p detection, qRT-PCR was performed by using the SYBR green PCR mix according to the manufacturer's instructions. The miR-423-5p and STMN1 expression level were normalized to the expression of $\mathrm{U} 6$ and GAPDH respectively. The relative mRNA expression was determined by the comparative Ct method. The primers were used as following: STMN1, Forward: 5'-GTACTTCTGGACTCACGGGC-3'; Reverse: 5'-AAGGCAAGAGTGGTCTGCTC-3'; GAPDH, Forward: 5'-TGAAGGTCGGAGTCAACGGA-3' Reverse: 5'-CCTGGAAGATGGTGATGGGAT-3'.

Western blot analysis

Total protein was extracted from cultured cell or tissue by using PIPA (200 m NaVO4, 2 X lysis buffer, $0.5 \mathrm{M}$ EDTA, $200 \mathrm{mM} \mathrm{NaF}, 10 \mathrm{X}$ sodiumdeoxycholate, $25 \mathrm{X}$ protease inhibitor) according to instructions. Total protein was separated by $12 \%$ SDS gel electrophoresis and then tranferred to the PVDF membranes (BioRad, USA). The membrane was probed the antibodies overnight (STMN1 and GAPDH, Abcam), then 


\section{Cellular Physiology Cell Physiol Biochem 2018;50:2249-2259

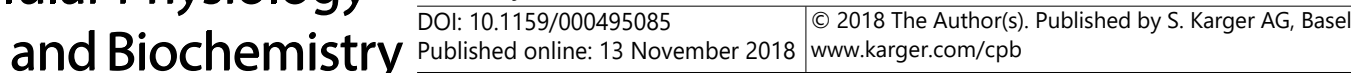 \\ Wang et al.: miR-423-5p Suppressed Osteosarcoma Proliferation and Invasion}

incubated with secondary antibodies. The signal of protein was detected with the chemiluminescence (Amersham Life Science, UK) following to the manufacturer's instructions. GAPDH was detected as the internal control protein.

\section{Cell Proliferation Assay, invasion and colony formation assay}

Cell Proliferation was accessed using the CCK-8 assay (Cell Counting Kit-8, Dojindo, Japan). Cells were cultured in the 96 -well plate after transfection. The number of cells was determined by measuring absorbance at the $450 \mathrm{~nm}$ on the microplate reader. Cell invasion was determined by using Transwell (Millipore Corporation, USA) coated with Matrigel (Life Technologies, USA). The MG-63 cells were cultured on the upper chamber in the DMEM with no-serum and 10\% FBS was added to the lower chamber. The invasive cells on the lower surface were fixed with methanol and stained with crystal violet. For cell colony formation, cells were kept on the 6-well plate (300 cells/well) and cultured for 2 weeks in the DMEM supplemented with $10 \%$ FBS. Cell colony was stained with crystal violet.

\section{Luciferase reporter assays}

The fragment of the wild type STMN1 3'UTR (WT 3'UTR) containing miR-423-5p target site was generated using PCR and the mutant STMN1 3'UTR (Mut 3'UTR) was amplified by PCR method. The fragments including the Mut 3'UTR or WT 3'UTR region of STMN1 were cloned into psiCHECK-2 vector (Promega, USA), which included firefly and renilla luciferase reporter gene. Cells were transfected with miR423-5p mimic and scramble and Mut 3'UTR or WT 3'UTR vector. Luciferase activity was determined by using the Dual-Luciferase Reporter analysis kit (Promega Corporation, USA) following to the instructions.

\section{Statistical analysis}

Data were presented as mean \pm SD (standard deviation). The difference between two groups was analyzed by Student's t-test and one-way analysis of variance was done to determine the difference when there were more than two groups. $\mathrm{P}<0.05$ was considered significantly different.

\section{Results}

miR-423-5p expression was decreased in osteosarcoma tissues and cell lines

We firstly detected the miR-423-5p expression in osteosarcoma tissues. As shown in the Fig. 1A, miR-423-5p expression was downregulated in 29 cases $(29 / 40 ; 72.5 \%)$ of osteosarcoma tissues compared with adjacent tissues. The expression of miR-423-5p was lower in osteosarcoma tissues than in the non-tumor specimens (Fig. 1B). We also showed that miR-423-5p expression was downregulated in osteosarcoma cell lines (U20S, SOSP9607, MG-63 and SAOS-2) and one normal osteoblast cell line (hFOB) (Fig. 1C).

\section{STMN1 expression was increased in osteosarcoma tissues and cell lines}

We next detected the STMN1 expression in the osteosarcoma tissues. As shown in the Fig. 2A, STMN1 expression was increased in 31 cases $(31 / 40 ; 77.5 \%)$ of osteosarcoma tissues compared with adjacent tissues. The expression of STMN1 was higher in osteosarcoma tissues than in the non-tumor specimens (Fig. 2B). We also demonstrated that the expression of STMN1 was upregulated in osteosarcoma cell lines (U20S, SOSP-9607, MG-63 and SAOS2) and one normal osteoblast cell line (hFOB) (Fig. 2C). Moreover, STMN1 expression was negatively correlated with miR-423-5p expression in osteosarcoma tissues (Fig. 2D).

\section{STMN1 was a direct target gene of miR-423-5p in osteosarcoma cell}

We transfected MG-63 cells with miR-423-5p mimic and found that the expression of miR-423-5p was significantly upregulated (Fig. 3A and B). TargetScan was used to search the candidate target genes of miR-423-5p. A conserved domain within the 3'-UTR of STMN1 contained a potential miR-423-5p binding site (Fig. 3C). The MG-63 cell was cotransfected 


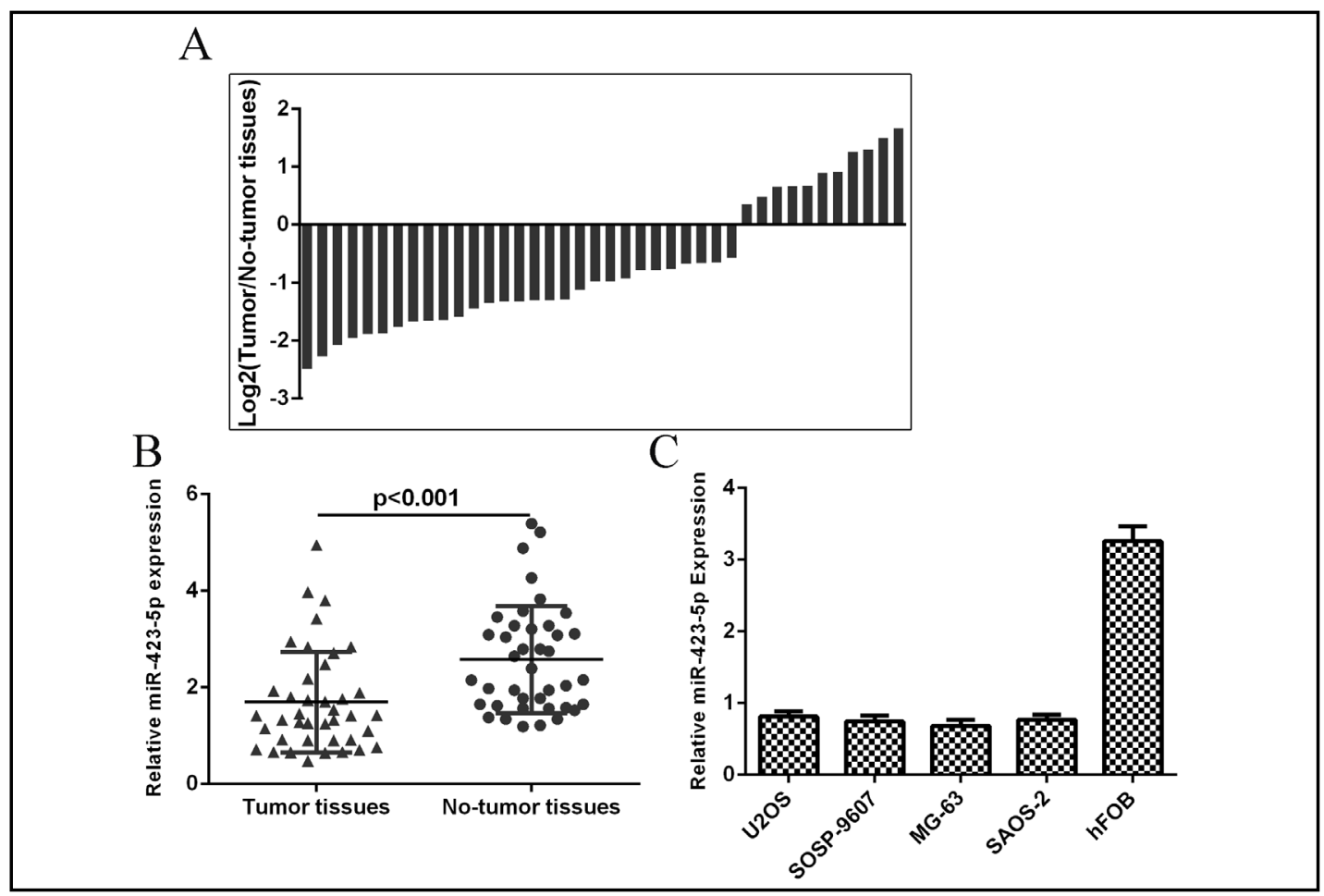

Fig. 1. miR-423-5p expression was downregulated in the osteosarcoma tissues and cell lines. (A) The miR423-5p expression was downregulated in 29 cases (29/40; $72.5 \%$ ) compared with adjacent tissues. Data are shown as log 2 of fold change of osteosarcoma tissues relative to non-tumor tissues. (B) The expression of miR-423-5p was lower in the osteosarcoma tissues than in the no-tumor specimens. (C) The expression of miR-423-5p in the osteosarcoma cell lines (U20S, SOSP-9607, MG-63 and SAOS-2) and one normal osteoblast cell line (hFOB) was measured by qRT-PCR.

with the mutated (MUT) and wild type (WT) STMN1 luciferase reporter together with miR-423-5p mimic or scramble. Overexpression of miR-423-5p suppressed the luciferase activity of WT reporter, but not the Mut reporter (Fig. 3D). Ectopic expression of miR-423-5p decreased STMN1 expression in the MG-63 cell (Fig. 3E).

STMN1 inhibited osteosarcoma cell proliferation, colony formation and invasion

Ectopic expression of miR-423-5p inhibited MG-63 cell proliferation (Fig. 4A). Elevated miR-423-5p expression decreased the expression of ki-67 in the MG-63 cell (Fig. 4B). Overexpression of miR-423-5p inhibited the expression of Cyclin D1 in MG-63 cell (Fig. 4C). Moreover, ectopic expression of miR-423-5p suppressed the MG-63 cell colony formation (Fig. 4D and E). Elevated miR-423-5p expression inhibited the MG-63 cell invasion (Fig. 4F and G).

STMN1 was involved in miR-423-5p-mediated cell behavior

qRT-PCR and Western blot assay disclosed that transfection with pCDNA3.1-STMN1 vector lead to an increase in STMN1 expression in MG-63 cells, compared with the control vector (Fig. 5A and B). STMN1 overexpression partially rescued the cell proliferation effect induced by miR-423-5p (Fig. 5C). Overexpression of STMN1 enhanced the expression of ki67 in the miR-423-5p-induced MG-63 cell (Fig. 5D). Ectopic expression of STMN1 enhanced the expression of Cyclin D1 in the miR-423-5p-induced MG-63 cell (Fig. 5E). Re-introduction of STMN1 significantly reversed the inhibition of cell colony formation in the miR-4235 p-expressing cells (Fig. 5F and G). Overexpression of STMN1 significantly enhanced the inhibition of cell invasion in the miR-423-5p-expressing cells (Fig. $5 \mathrm{H}$ and I). 


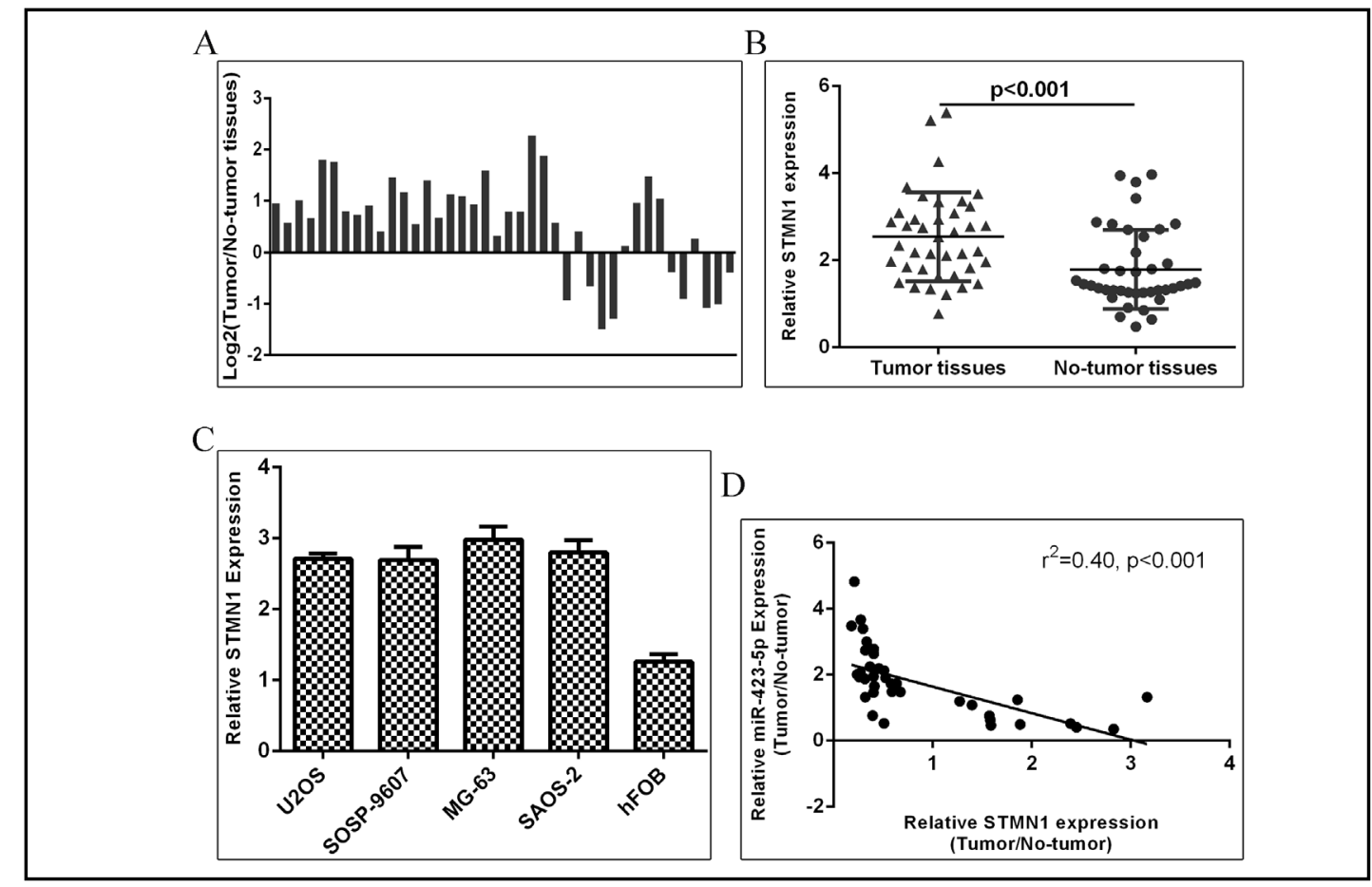

B

Fig. 2. STMN1 expression was upregulated in the osteosarcoma tissues and cell lines. (A) The STMN1 expression was upregulated in 31 cases (31/40; $77.5 \%$ ) compared with adjacent tissues. Data are shown as $\log 2$ of fold change of osteosarcoma tissues relative to non-tumor tissues. (B) The expression of STMN1 was higher in the osteosarcoma tissues than in the no-tumor specimens. (C) The expression of STMN1 in the osteosarcoma cell lines (U20S, SOSP-9607, MG-63 and SAOS-2) and one normal osteoblast cell line (hFOB) was measured by qRT-PCR. (D) The expression of STMN1 was negatively related with the expression of miR423-5p in the osteosarcoma tissues.

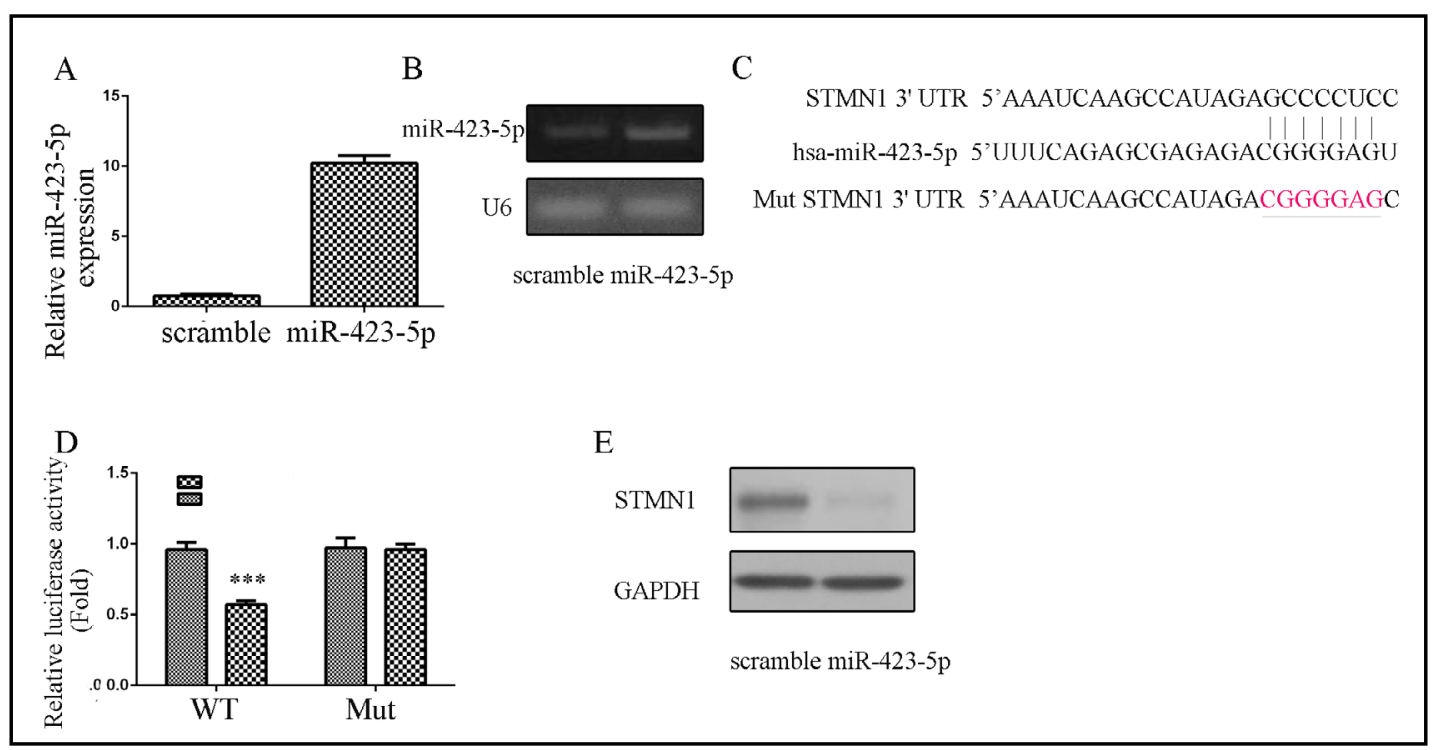

Fig. 3. STMN1 was a direct target gene of miR-423-5p in the osteosarcoma cell. (A) The expression of miR423-5p was determined by qRT-PCR. (B) The expression of miR-423-5p was determined by PCR. (C) A conserved domain within the 3'-UTR of STMN1 with a potential miR-423-5p binding site was shown. (D) Overexpression of miR-423-5p suppressed the luciferase activity of WT reporter, but not the Mut reporter. (E) Ectopic expression of miR-423-5p inhibited the expression of STMN1 in the MG-63 cell. ***p<0.001. 


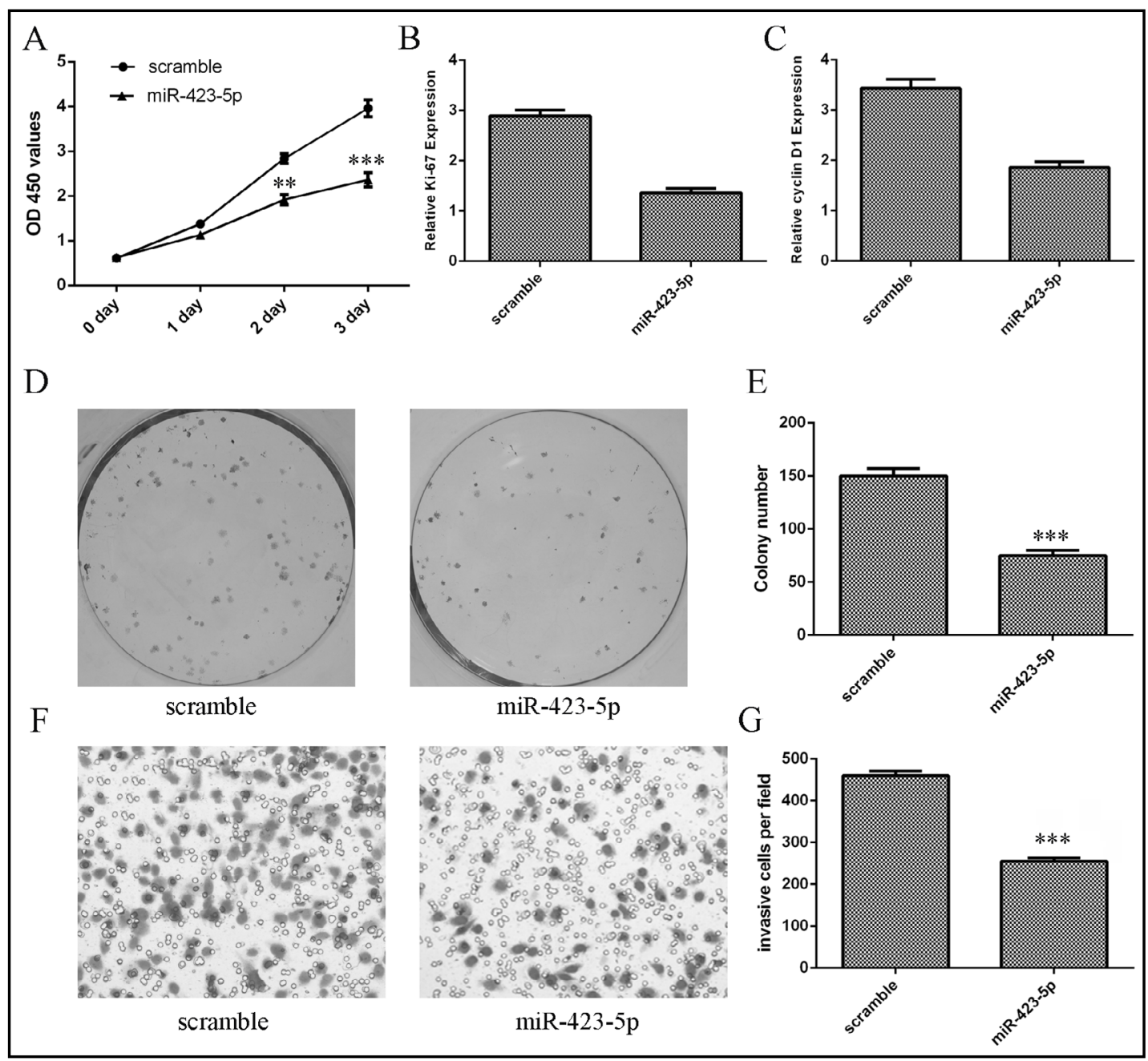

Fig. 4. STMN1 suppressed the osteosarcoma cell proliferation, colony formation and invasion. (A) Ectopic expression of miR-423-5p suppressed the MG-63 cell proliferation. (B) Elevated miR-423-5p expression decreased the ki-67 expression in the MG-63 cell. (C) Overexpression of miR-423-5p inhibited the cyclin D1 expression in the MG-63 cell. (D) Ectopic expression of miR-423-5p suppressed the MG-63 cell colony formation. (E) The relative colony numbers were shown. (F) Elevated miR-423-5p expression inhibited the MG-63 cell invasion. (G) The relative invasive cells were shown. ${ }^{* *} \mathrm{p}<0.01$ and ${ }^{* * *} \mathrm{p}<0.001$. 


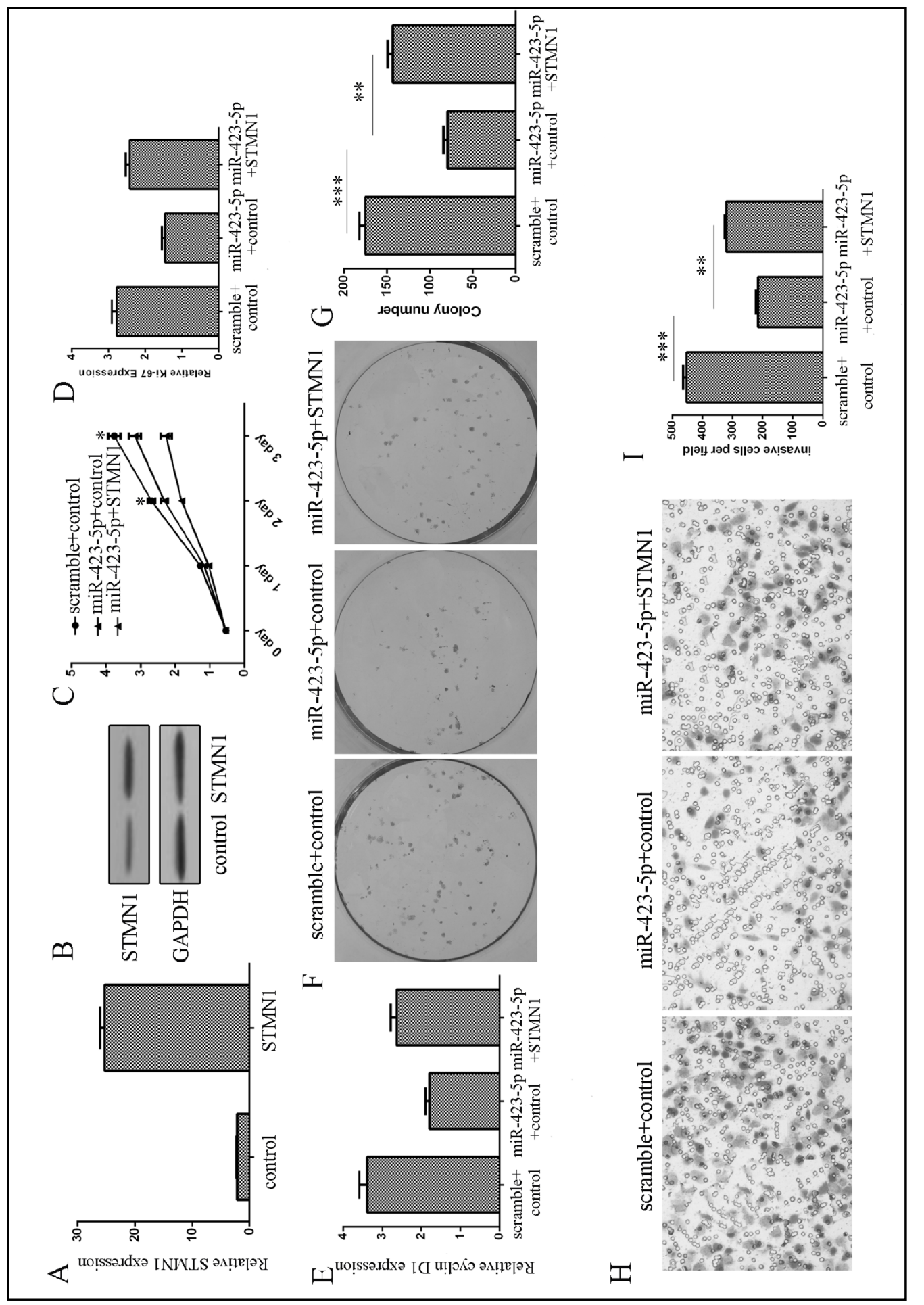

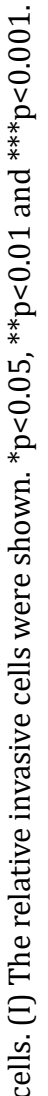

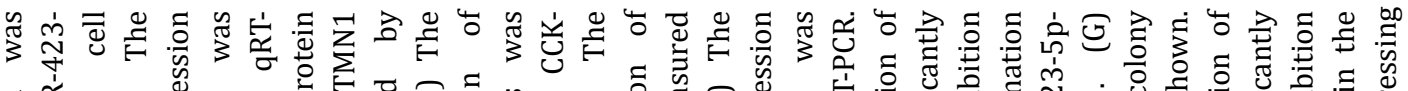

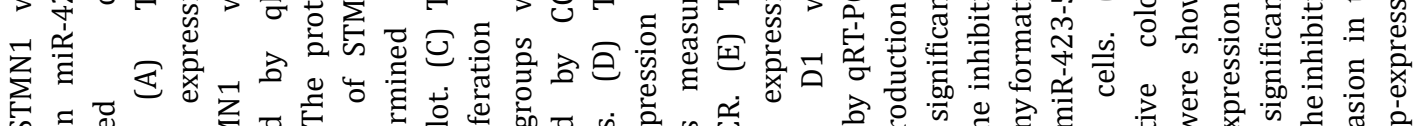

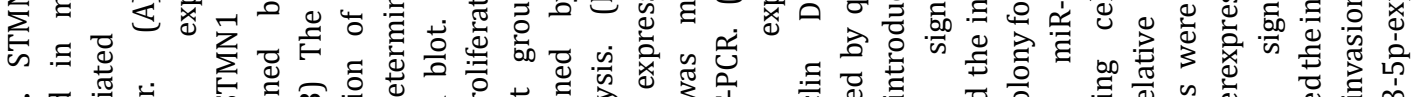

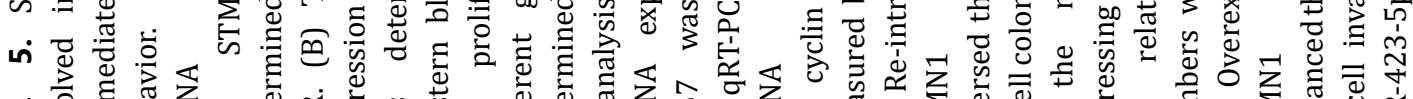

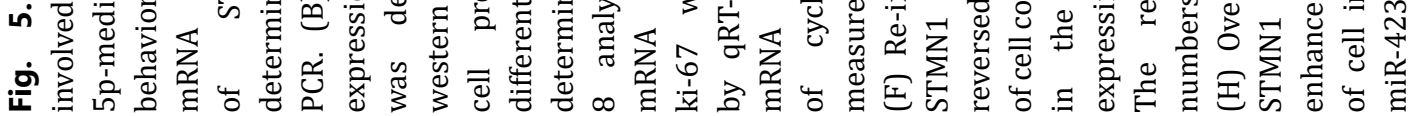
KARGER 


\section{Cellular Physiology Cell Physiol Biochem 2018;50:2249-2259

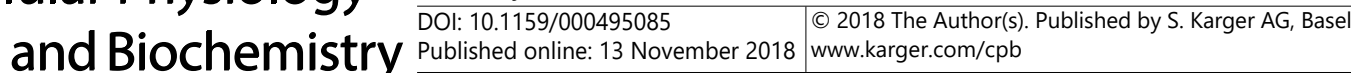 \\ Wang et al.: miR-423-5p Suppressed Osteosarcoma Proliferation and Invasion}

\section{Discussion}

Although several miRNAs have been found to be deregulated in osteosarcoma cells and tissues, the exact mechanism by how miRNA regulated the tumorigenesis is still not fully elucidated [31-34]. Our study showed that miR-423-5p expression was decreased in osteosarcoma tissues and cell lines. The expression of STMN1 was upregulated in osteosarcoma tissues and cell lines. Moreover, STMN1 expression was negatively related with miR-423-5p expression in osteosarcoma tissues. We identified STMN1 as a direct target gene of miR-423-5p in osteosarcoma cell. Overexpression of miR-423-5p suppressed osteosarcoma cell proliferation, colony formation and invasion. Furthermore, we demonstrated that STMN1 was involved in miR-423-5p-mediated cell behavior such as cell proliferation, colony formation and invasion in osteosarcoma cell. Our research indicated that miR-423-5p acted as a tumor suppressor gene in osteosarcoma partly through inhibiting STMN1 expression.

Previous studies revealed that miR-423-5p acted significant roles in the development of tumors [35-37]. For example, Li et al. [38] showed that miR-423-5p expression was increased in gliomas tissues and ecoptic expression of miR-423-5p increased glioma cell angiogenesis, proliferation and invasion through targeting ING-4. Lu et al. [39] demonstrated that serum miR-423-5p was upregulated in the stage I-II colorectal cancer patients compared with the control. Liu et al. demonstrated that miR-423-5p increased cell proliferation and invasion through regulating trefoil factor 1 (TFF1) in gastric cancer cells [40]. However, the expression and effect of miR-423-5p are still unknown in osteosarcoma. Our study showed that miR423-5p expression was decreased in osteosarcoma tissues. The miR-423-5p expression was downregulated in 29 cases $(29 / 40 ; 72.5 \%)$ of osteosarcoma tissues compared with the adjacent tissues. Furthermore, we indicated that the expression of miR-423-5p was upregulated in osteosarcoma cell lines (U20S, SOSP-9607, MG-63 and SAOS-2) and one normal osteoblast cell line (hFOB). Moreover, overexpression of STMN1 suppressed the osteosarcoma cell proliferation, colony formation and invasion. Our results demonstrated that miR-423-5p acted as a tumor suppressor gene in osteosarcoma.

Next, we examined the molecular mechanism about the function of miR-423-5p and demonstrated that STMN1 was a target for miR-423-5p in the osteosarcoma cell. STMN1 is a $17-\mathrm{kDa}$ cytoplasmic protein, which is also named as oncoprotein 18 (OP18) [4143]. Previous research showed that STMN1 acted as an important regulator in cell cycle, proliferation, invasion and survival through regulating microtubule dynamics [44-46]. STMN1 was upregulated in several human tumors such as lung cancer, gastric cancer, oral squamous-cell carcinoma, colorectal tumor, gallbladder cancer and breast cancer [4751]. Our data identified STMN1 as a direct target gene of miR-423-5p in osteosarcoma cell. TargetScan system showed that there was a conserved domain within the 3'-UTR of STMN1 with a potential miR-423-5p binding site. Luciferase reporter assay demonstrated that ectopic expression of miR-423-5p suppressed the luciferase activity of WT reporter, but not the Mut reporter. Overexpression of miR-423-5p suppressed the expression of STMN1 in the MG-63 cell. Furthermore, we demonstrated thatSTMN1 expression was increased in osteosarcoma tissues and cell lines. STMN1 expression was negatively corelated with miR423-5p expression in osteosarcoma tissues. In addition, STMN1 was demonstrated to be involved in miR-423-5p-mediated cell behavior such as cell proliferation, colony formation and invasion in the osteosarcoma cell.

\section{Conclusion}

We found that miR-423-5p expression was downregulated in osteosarcoma tissues and cell lines. Overexpression of miR-423-5p inhibited osteosarcoma cell proliferation, colony formation and invasion through suppressing STMN1 expression. Our data suggested that miR-423-5p acted as a tumor suppressor gene in osteosarcoma partly through inhibiting the expression of STMN1. 


\section{Cellular Physiology Cell Physiol Biochem 2018;50:2249-2259

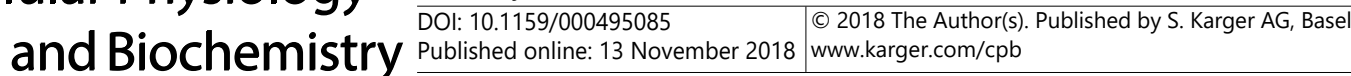 \\ Wang et al.: miR-423-5p Suppressed Osteosarcoma Proliferation and Invasion}

\section{Disclosure Statement}

The authors declare no conflicts of interest.

\section{References}

1 Xing B, Ren C: Tumor-suppressive miR-99a inhibits cell proliferation via targeting of TNFAIP8 in osteosarcoma cells. Am J Transl Res 2016;8:1082-1090.

2 Yu Z, Zhang Y, Gao N, Wang X: Overexpression of miR-506 inhibits growth of osteosarcoma through Snail2. Am J Transl Res 2015;7:2716-2723.

3 Niu G, Li B, Sun J, Sun L: miR-454 is down-regulated in osteosarcomas and suppresses cell proliferation and invasion by directly targeting c-Met. Cell Prolif 2015;48:348-355.

4 Senerchia AA, Macedo CR, Ferman S, Scopinaro M, Cacciavillano W, Boldrini E, de Moraes VLL, Rey G, de Oliveira CT, Castillo L, Almeida MT, Borsato ML, Lima E, Lustosa D, Barreto JH, El-Jaick T, Aguiar S, Brunetto A, Greggiani L, Cogo-Moreira H et al.: Results of a Randomized, Prospective Clinical Trial Evaluating Metronomic Chemotherapy in Nonmetastatic Patients With High-Grade, Operable Osteosarcomas of the Extremities: A Report From the Latin American Group of Osteosarcoma Treatment. Cancer 2017;123:10031010.

5 Lu K, Su S, Lin C, Hsieh Y, Lin Y, Chien M, Reiter R, Yang S: Melatonin attenuates osteosarcoma cell invasion by suppression of $\mathrm{C}$-C motif chemokine ligand 24 through inhibition of the c-Jun N-terminal kinase pathway. J. Pineal Res. 2018;e12507.

6 Yin Z, Ding H, He E, Chen J, Li M: Up-regulation of microRNA-491-5p suppresses cell proliferation and promotes apoptosis by targeting FOXP4 in human osteosarcoma. Cell Prolif 2016;10.1111/cpr.12308.

7 Wang Y, Jia LS, Yuan W, Wu Z, Wang HB, Xu T, Sun JC, Cheng KF, Shi JG: Low miR-34a and miR-192 are associated with unfavorable prognosis in patients suffering from osteosarcoma. Am J Transl Res 2015;7:111-119.

-8 Gao Y, Luo LH, Li S, Yang C: miR-17 inhibitor suppressed osteosarcoma tumor growth and metastasis via increasing PTEN expression. Biochem Biophys Res Commun 2014;444:230-234.

-9 Niu G, Li B, Sun L, An C: MicroRNA-153 Inhibits Osteosarcoma Cells Proliferation and Invasion by Targeting TGF-beta2. PLoS One 2015;10:e0119225.

10 Dai HZ, Lv YF, Yan GN, Meng G, Zhang X, Guo QN: RanBP9/TSSC3 complex cooperates to suppress anoikis resistance and metastasis via inhibiting Src-mediated Akt signaling in osteosarcoma. Cell Death Dis 2016; 7:e2572.

11 Tang J, Shen L, Yang Q, Zhang C: Overexpression of metadherin mediates metastasis of osteosarcoma by regulating epithelial-mesenchymal transition. Cell Prolif 2014;47:427-434.

12 Han K, Chen X, Bian N, Ma B, Yang T, Cai C, Fan Q Zhou Y, Zhao TB: MicroRNA profiling identifies MiR-195 suppresses osteosarcoma cell metastasis by targeting CCND1. Oncotarget 2015;6:8875-8889.

13 Salah Z, Arafeh R, Maximov V, Galasso M, Khawaled S, Abou-Sharieha S, Volinia S, Jones KB, Croce CM, Aqeilan RI: miR-27a and miR-27a* contribute to metastatic properties of osteosarcoma cells. Oncotarget 2015;6:4920-4935.

14 Huang K, Dong X, Sui C, Hu D, Xiong T, Liao S, Zhang H: MiR-223 suppresses endometrial carcinoma cells proliferation by targeting IGF-1R. Am J Transl Res 2014;6:841-849.

15 Ahmad A, Sethi S, Chen W, Ali-Fehmi R, Mittal S, Sarkar FH: Up-regulation of microRNA-10b is associated with the development of breast cancer brain metastasis. Am J Transl Res 2014;6:384-390.

16 Liang J, Liu X, Xue H, Qiu B, Wei B, Sun K: MicroRNA-103a inhibits gastric cancer cell proliferation, migration and invasion by targeting c-Myb. Cell Prolif 2015;48:78-85.

17 Qiu Y, Yu H, Shi X, Xu K, Tang Q, Liang B, Hu S, Bao Y, Xu J, Cai J, Peng W, Cao Q, Yin P: microRNA-497 inhibits invasion and metastasis of colorectal cancer cells by targeting vascular endothelial growth factor-A. Cell Prolif 2016;49:69-78.

18 Shi Z, An N, Lu BM, Zhou N, Yang SL, Zhang B, Li CY, Wang ZJ, Wang F, Wu CF, Bao JK: Identification of novel kinase inhibitors by targeting a kinase-related apoptotic protein-protein interaction network in HeLa cells. Cell Prolif 2014;47:219-230. 


\section{Cellular Physiology Cell Physiol Biochem 2018;50:2249-2259

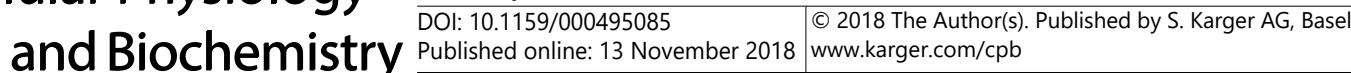 \\ Wang et al.: miR-423-5p Suppressed Osteosarcoma Proliferation and Invasion}

19 Lu D, Davis MP, Abreu-Goodger C, Wang W, Campos LS, Siede J, Vigorito E, Skarnes WC, Dunham I, Enright AJ, Liu P: MiR-25 regulates Wwp2 and Fbxw7 and promotes reprogramming of mouse fibroblast cells to iPSCs. PLoS One 2012;7:e40938.

20 Kalman S, Garbett KA, Vereczkei A, Shelton RC, Korade Z, Mirnics K: Metabolic stress-induced microRNA and mRNA expression profiles of human fibroblasts. Exp Cell Res 2014;320:343-353.

21 Tsukerman P, Stern-Ginossar N, Gur C, Glasner A, Nachmani D, Bauman Y, Yamin R, Vitenshtein A, Stanietsky N, Bar-Mag T, Lankry D, Mandelboim O: MiR-10b downregulates the stress-induced cell surface molecule MICB, a critical ligand for cancer cell recognition by natural killer cells. Cancer Res 2012;10.1158/0008-5472.CAN-11-2671.

-22 Yu L, Ding GF, He C, Sun L, Jiang Y, Zhu L: MicroRNA-424 is down-regulated in hepatocellular carcinoma and suppresses cell migration and invasion through c-Myb. PLoS One 2014;9:e91661.

23 Wang F, Xiao W, Sun J, Han D, Zhu Y: MiRNA-181c inhibits EGFR-signaling-dependent MMP9 activation via suppressing Akt phosphorylation in glioblastoma. Tumour Biol 2014;35:8653-8658.

24 Toiyama Y, Okugawa Y, Goel A: DNA methylation and microRNA biomarkers for noninvasive detection of gastric and colorectal cancer. Biochem Biophys Res Commun 2014;10.1016/j.bbrc.2014.08.001.

25 Wu DD, Li XS, Meng XN, Yan J, Zong ZH: MicroRNA-873 mediates multidrug resistance in ovarian cancer cells by targeting ABCB1. Tumour Biol 2016;10.1007/s13277-016-4944-y.

-26 Wang RJ, Li JW, Bao BH, Wu HC, Du ZH, Su JL, Zhang MH, Liang HQ: MiRNA-873 Inhibits Glioblastoma Tumorigenesis and Metastasis by Suppressing the Expression of IGF2BP1. J Biol Chem 2015;10.1074/jbc. M114.624700.

27 Zheng H, Zhang F, Lin X, Huang C, Zhang Y, Li Y, Lin J, Chen W: MicroRNA-1225-5p inhibits proliferation and metastasis of gastric carcinoma through repressing insulin receptor substrate-1 and activation of betacatenin signaling. Oncotarget 2016;7:4647-4663.

28 Huang YZ, Zhang J, Shao HY, Chen JP, Zhao HY: MicroRNA-191 promotes osteosarcoma cells proliferation by targeting checkpoint kinase 2. Tumour Biol 2015;10.1007/s13277-015-3290-9.

29 Du JY, Wang LF, Wang Q Yu LD: miR-26b inhibits proliferation, migration, invasion and apoptosis induction via the downregulation of 6-phosphofructo-2-kinase/fructose-2, 6-bisphosphatase-3 driven glycolysis in osteosarcoma cells. Oncol Rep 2015;33:1890-1898.

- 30 Xu JQ, Zhang WB, Wan R, Yang YQ: MicroRNA-32 inhibits osteosarcoma cell proliferation and invasion by targeting Sox9. Tumour Biol 2014;10.1007/s13277-014-2229-x.

31 Xu H, Liu X, Zhao J: Down-regulation of miR-3928 promoted osteosarcoma growth. Cell Physiol Biochem 2014;33:1547-1556.

32 Wang XH, Cai P, Wang MH, Wang Z: microRNA25 promotes osteosarcoma cell proliferation by targeting the cellcycle inhibitor p27. Mol Med Rep 2014;10:855-859.

-33 Wang J, Xu G, Shen F, Kang Y: miR-132 targeting cyclin E1 suppresses cell proliferation in osteosarcoma cells. Tumour Biol 2014;35:4859-4865.

-34 Tian Y, Zhang YZ, Chen W: MicroRNA-199a-3p and microRNA-34a regulate apoptosis in human osteosarcoma cells. Biosci Rep 2014;34:doi:10.1042/BSR20140084.

-35 Zhao H, Gao A, Zhang Z, Tian R, Luo A, Li M, Zhao D, Fu L, Dong JT, Zhu Z: Genetic analysis and preliminary function study of miR-423 in breast cancer. Tumour Biol 2015;36:4763-4771.

-36 Morales S, Gulppi F, Gonzalez-Hormazabal P, Fernandez-Ramires R, Bravo T, Reyes JM, Gomez F, Waugh E, Jara L: Association of single nucleotide polymorphisms in Pre-miR-27a, Pre-miR-196a2, Pre-miR-423, miR-608 and Pre-miR-618 with breast cancer susceptibility in a South American population. BMC Genet 2016;17:109.

37 Ma Y, Wang R, Zhang J, Li W, Gao C, Liu J, Wang J: Identification of miR-423 and miR-499 polymorphisms on affecting the risk of hepatocellular carcinoma in a large-scale population. Genet Test Mol Biomarkers 2014;18:516-524.

-38 Li S, Zeng A, Hu Q Yan W, Liu Y, You Y: miR-423-5p contributes to a malignant phenotype and temozolomide chemoresistance in glioblastomas. Neuro Oncol 2017;19:55-65.

-39 Lu X, Lu J: The significance of detection of serum miR-423-5p and miR-484 for diagnosis of colorectal cancer. Clin Lab 2015;61:187-190.

40 Liu J, Wang X, Yang X, Liu Y, Shi Y, Ren J, Guleng B: miRNA423-5p regulates cell proliferation and invasion by targeting trefoil factor 1 in gastric cancer cells. Cancer Lett 2014;347:98-104. 


\section{Cellular Physiology Cell Physiol Biochem 2018;50:2249-2259

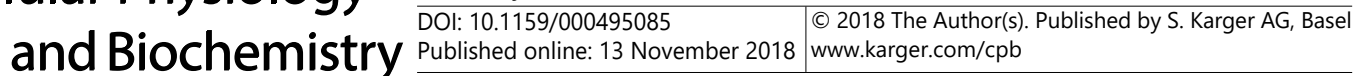

41 Dou YD, Zhao H, Huang T, Zhao SG, Liu XM, Yu XC, Ma ZX, Zhang YC, Liu T, Gao X, Li L, Lu G, Chan WY, Gao F, Liu HB, Chen ZJ: STMN1 Promotes Progesterone Production Via StAR Up-regulation in Mouse Granulosa Cells. Sci Rep 2016;6:26691.

42 Zhang HQ Guo X, Guo SQ Wang Q Chen XQ, Li XN, Guo LS: STMN1 in colon cancer: expression and prognosis in Chinese patients. Eur Rev Med Pharmacol Sci 2016;20:2038-2044.

-43 Li J, Hu G, Kong F, Wu K, Song K, He J, Sun W: Elevated STMN1 Expression Correlates with Poor Prognosis in Patients with Pancreatic Ductal Adenocarcinoma. Pathol Oncol Res 2015;21:1013-1020.

$>44$ Li J, Kong F, Wu K, Song K, He J, Sun W: miR-193b directly targets STMN1 and uPA genes and suppresses tumor growth and metastasis in pancreatic cancer. Mol Med Rep 2014;10:2613-2620.

-45 Li J, Hu GH, Kong FJ, Wu KM, He B, Song K, Sun WJ: Reduced STMN1 expression induced by RNA interference inhibits the bioactivity of pancreatic cancer cell line Panc-1. Neoplasma 2014;61:144-152.

$\checkmark 46$ Wu N, Gao N, Fan D, Wei J, Zhang J, An J: miR-223 inhibits dengue virus replication by negatively regulating the microtubule-destabilizing protein STMN1 in EAhy926 cells. Microbes Infect 2014;16:911-922.

-47 Zou ZQ, Du YY, Sui G, Xu SN: Expression of TS, RRM1, ERCC1, TUBB3 and STMN1 Genes in Tissues of Nonsmall Cell Lung Cancer and its Significance in Guiding Postoperative Adjuvant Chemotherapy. Asian Pac J Cancer Prev 2015;16:3189-3194.

-48 Kouzu Y, Uzawa K, Koike H, Saito K, Nakashima D, Higo M, Endo Y, Kasamatsu A, Shiiba M, Bukawa H, Yokoe H, Tanzawa H: Overexpression of stathmin in oral squamous-cell carcinoma: correlation with tumour progression and poor prognosis. Br J Cancer 2006;94:717-723.

49 Cao Y, Zhang G, Wang P, Zhou J, Gan W, Song Y, Huang L, Zhang Y, Luo G, Gong J, Zhang L: Clinical significance of UGT1A1 polymorphism and expression of ERCC1, BRCA1, TYMS, RRM1, TUBB3, STMN1 and TOP2A in gastric cancer. BMC Gastroenterol 2017;17:2.

-50 Wang J, Yao Y, Ming Y, Shen S, Wu N, Liu J, Liu H, Suo T, Pan H, Zhang D, Ding K: Downregulation of stathmin 1 in human gallbladder carcinoma inhibits tumor growth in vitro and in vivo. Sci Rep 2016;6:28833.

-51 Kuang XY, Jiang HS, Li K, Zheng YZ, Liu YR, Qiao F, Li S, Hu X, Shao ZM: The phosphorylation-specific association of STMN1 with GRP78 promotes breast cancer metastasis. Cancer Lett 2016;377:87-96. 